\title{
Zum Einfluss affektiver Faktoren auf den frühen Fremdsprachenerwerb im Elementarbereich durch den narrativen Ansatz
}

\author{
The influence of affective factors on early foreign \\ language acquisition at elementary level according \\ to the storytelling approach
}

ABSTRACT. The article presents the impact of emotions on teaching children foreign languages. To this end, the results of a research project carried out under the auspices of the Institute of German Philology at the University of Silesia in Katowice are discussed. The project consisted in providing language courses at three kindergartens and one primary school. During those courses, German was taught as a foreign language using the storytelling approach. The project results led to the conclusion that, unlike traditional teaching methods, which are based on very limited input and intensive imitation, the teaching method used creates much more favourable conditions for the activation of innate language acquiring processes and takes the level of the children's cognitive development into account to a larger degree. The objectives of the evaluation were - among other things - to determine how the storytelling approach affects children's attitudes to a foreign language, whether it arouses their internal motivation for acquiring a foreign language, whether it contributes to building their confidence, and whether it stimulates their imagination and creativity.

KEYWORDS: storytelling approach, emotions, motivation, early school education, language input, stories, affective filter.

SCHLÜSSELWORTE: narrativer Ansatz, Emotionen, Motivation, Frühunterricht, sprachlicher Input, Geschichten, affektiver Filter. 


\section{EINLEITUNG}

Emotionen begleiten und beeinflussen das menschliche Denken, Wahrnehmen, Handeln und Sprechen; sie lösen Reaktionen auf wahrgenommene Reize, wie z.B. Staunen, Stolz, Wut, Freude, Langeweile aus und wirken sich auf die sozialen Beziehungen zwischen Gesprächspartnern aus, indem sie die Dynamik unserer Zuwendung bzw. Abwendung gegenüber der umgebenden Welt determinieren (Iluk 2002a: 133-134; Iluk 2002b: 96). Emotionen sind laut Ciompi (1997: 95-99):

Die Energielieferanten oder ,Motoren' und ,Motivatoren' aller kognitiven Dynamik, (...) bestimmen andauernd den Fokus der Aufmerksamkeit, (...) wirken wie Schleusen oder Pforten, die den Zugang zu unterschiedlichen Gedächtnisspeichern öffnen oder schließen, (...) bestimmen die Hierarchie unserer Denkinhalte.

Die Bedeutung affektiver Komponenten in Wahrnehmung und Erleben wird mittlerweile auch beim Erlernen einer Fremdsprache gesehen und findet somit Eingang in die handlungsorientierte Fremdsprachendidaktik. Leopold-Mudrack (1998: 57) vertritt die Auffassung, dass alle von einer Emotion begleiteten Dinge besser als rein abstrakte Wörter abgespeichert werden, die in keinen Kontext eingebettet sind oder in keinem Zusammenhang mit dem Lerner selbst stehen. Daher ist es im Sinne einer ganzheitlichen Fremdsprachenvermittlung unerlässlich, insbesondere junge Lernende affektiv anzusprechen und ihre Emotionen in den Lernprozess mit einzubeziehen. Die ersten Wochen bzw. Monate bestimmen in besonderem Maße die sich anbahnende habituelle Motivation zum Sprachlernen sowie eine positive (oder manchmal negative) Einstellung zum Gegenüber.

Im vorliegenden Beitrag wird der Versuch unternommen, den Zusammenhang zwischen Emotionen und Fremdsprachenlernen im Kindergarten und Primarbereich genauer zu beleuchten. Zu diesem Zweck werden die Ergebnisse eines eigens durchgeführten Forschungsprojektes dargestellt und kommentiert, in dem der Frage nachgegangen wird, ob der narrative Ansatz als die eingesetzte Unterrichtsform zu Lernfortschritten und authentischen Lernerfolgen beiträgt. Außerdem gilt es zu ergründen, ob junge Lernenden dabei positive Emotionen und sichtliche Lernfreude erleben, die die Motivation, die Aufmerksamkeit, das Behalten und die Kreativität beim frühen Fremdsprachenerwerb wesentlich fördern. 


\section{PRINZIPIEN DES FRÜHEN FREMDSPRACHENUNTERRICHTS}

In den nativistischen Lerntheorien wird davon ausgegangen, dass die Fähigkeit zum Sprachenlernen angeboren ist und jedes Kind über einen natürlichen Spracherwerbsmechanismus verfügt. Diese natürlichen Spracherwerbsfähigkeiten werden durch den Input aktiviert, der an Lernende herangetragen wird (Weskamp 2003: 25). Im Sinne von Krashen (1985) und Vogel (1993) sollte optimaler, qualitativ hochwertiger sprachlicher Input für die Lernenden verständlich sein, da ansonsten die Informationsverarbeitungsprozesse entweder völlig blockiert oder überhaupt nicht in Gang gesetzt werden können. Außerdem darf der Input keiner grammatischen Progression folgen, muss im ausreichenden Maße vorhanden sein, sprachlich und phonetisch korrekt sowie interessant und persönlich relevant sein (Legutke, Schocker-von Ditfurth, Müller-Hartmann 2013: 291; Lundquist-Mog, Widlok 2015: 26). Iluk (2005: 171) stimmt der These $\mathrm{zu}$, dass inhaltlich interessante Lernmaterialien die emotionale Seite des Lerners ansprechen, sodass die Informationsaufnahme und -verarbeitung wesentlich unterstützt und eine Grundlage für eine stärkere Motivation zum Fremdsprachenlernen geschaffen werden kann. Der "affektive Filter ${ }^{\prime \prime} 1$ ist dann weit geöffnet und blockiert nicht den Fluss der Informationen $\mathrm{zu}$ den kognitiven Zentren, wo sie verarbeitet und memoriert werden. Wenn dagegen den Kindern Inhalte angeboten werden, die Monotonie und die intellektuelle Unterforderung bewirken, wie z.B. fremdsprachige Etiketten für bekannte Begriffe ( $a$ dog - two dogs, a book - two books usw.), so kann das zu völligem Desinteresse am Fremdsprachenunterricht oder sogar zum Lernabbruch führen (Leopold-Mudrack 1998: 127; Iluk 2005: 164).

Die Forderung, dass die Lernenden von Anfang an mit authentischen Sprachdaten konfrontiert werden sollten, geht mit einer interessensgeleiteten Auswahl einher:

Die Schulkinder sollten mit der L2 in ihrer „natürlichen“ Form konfrontiert werden und nicht mit einem reduzierten Input, aus dem alles herausgefiltert wurde, was noch nicht im Unterricht behandelt wurde - vorausgesetzt natürlich, es ist ihrem Alter angemessen und vermittelt Inhalte, die ihren Interessen entgegenkommen. Die Auswahl der , bearbeiteten' Strukturen innerhalb dieses Inputs kann den impliziten Lernvorgängen überlassen bleiben (Bleyhl 2003: 7).

Darüber hinaus sollte den Kindern ein uneingeschränkter Kontakt mit der Zielsprache gewährleistet werden, weil nur dann der natürliche Spracherwerbsmechanismus funktionieren kann. Dementsprechend muss ein in-

\footnotetext{
${ }^{1}$ Die Theorie des affektiven Filters von Krashen erklärt die Auswirkungen von Emotionen vor allem auf den unbewussten Fremdsprachenerwerb und lässt die affektiven Aspekte des Lernprozesses erfassen (Iluk 2006a: 274).
} 
tensiver und reichhaltiger Input angeboten werden, damit die Kinder intuitiv Gesetzmäßigkeiten der fremden Sprache entdecken können (Gładysz 2007: 228; Lundquist-Mog, Widlok 2015: 26).

Im Kontext des frühen Fremdsprachenlernens ist darüber hinaus auf ausreichende Methodenvielfalt und Übungswechsel unter Berücksichtigung von Bewegungsphasen zu achten. Nach einer Phase der Konzentration sollte im Sinne von Leopold-Mudrack (1998: 61) sowie Lundquist-Mog und Widlok (2015: 123) eine Phase der Bewegung erfolgen, in der die Kinder die Gelegenheit haben, neue Kraft zu schöpfen. Aus neurowissenschaftlicher Sicht werden Lerninhalte, die in Verbindung mit Bewegung angeeignet werden, für einen längeren Zeitraum gefestigt und neuronal vernetzt. Vom effektiven Lernen ist dann zu sprechen, wenn die Lernenden selbst aktiv sind, sich bewegen und handeln (Leopold-Mudrack 1998: 52; Lundquist-Mog, Widlok 2015: 123). Erwähnung finden sollte hier das Konzept der so genannten bewegten Schule mit dem Motto: „Bewegung ist das Tor zum Lernen“, das die Gehirngymnastik mit einbezieht und in Deutschland an Grundschulen verbreitet ist:

Vor allem zwei Grundannahmen bestimmen dies Konzept: Zum einen wird angenommen, dass Lernen bloß effizient sein kann, wenn beide Gehirnhälften von Menschen in gleicher Weise aktiviert und dabei aufeinander bezogen werden. Hier greift es auf neurodidaktische Vorstellungen über ganzheitliches Lernen zurück, nach denen es darauf ankommt beide Gehirnhemisphären in einem stets ausgewogenen Verhältnis zueinander in Lernaktivitäten zu versetzen. Zum zweiten geht es davon aus, dass solche Gehirnbalance vorzüglich dadurch erreicht werden kann, dass äußere körperliche Bewegungen angestellt werden. Dabei geht es aber nicht um irgendwelche körperlichen Bewegungen, sondern vor allem um solche, bei denen Arme und Beine von Lernenden ÜberkreuzBewegungen vollziehen. Man spricht auch vom Kreuzdiagonalmuster der Bewegungen (Peterßen 1999: 50).

Leopold-Mudrack (1998: 52) vertritt die Ansicht, dass die Bewegung einen wesentlichen Beitrag zur gesamten Entwicklung des Kindes leistet. Dadurch lassen sich das Vorstellungsvermögen, die Emotionalität sowie die kindliche Phantasie fördern.

\section{ROLLE DER EMOTIONEN IM FRÜHEN FREMDSPRACHENLERNEN UND -LEHREN}

Als ein primäres Ziel des frühen Fremdsprachenlehrens gilt es, Freude und Motivation für das Lernen und den Gebrauch der fremden Sprache zu wecken und längerfristig aufrechtzuerhalten (Legutke et al. 2013: 291). Fak- 
toren wie Selbstvertrauen, geringe gefühlsmäßige Hemmungen und der Wunsch nach Integration tragen entscheidend zum Erfolg des Fremdsprachenerwerbs bei. Ein Lerner mit positivem Selbstverständnis bemüht sich darum, mehr Input zu erlangen und nimmt ihn offener auf. Bei gleichem Input nimmt der Lerner mit den geringeren gefühlsmäßigen Hemmungen mehr auf als der Lerner mit den größeren. Die besondere Rolle von Emotionen im Unterricht betont Iluk (2006a: 276):

Negative Emotionen, wie etwa Hoffnungslosigkeit oder Langeweile, besonders bei mehrfach erlebtem Misserfolg, machen jedes weitere effiziente Lernen unmöglich. Dagegen Emotionen positiver Art, wie etwa Freude, Lust, Spaß, Stolz, Erfolgsgefühl, fördern eine kreative Informationsverarbeitung, flüssigeres, kreativeres, besseres Erinnern und schnelleres Problemlösen.

Im Fremdsprachenunterricht sollte darauf geachtet werden, dass immer wieder positive Emotionen bei den Lernenden ausgelöst werden, denn

entuzjazm i emocje pokrewne, jak fascynacja, ciekawość, radość, głód wiedzy są - zdaniem neurobiologów - najważniejszymi czynnikami napędowymi dla mózgu, ponieważ aktywizują centra emocjonalne, które, pobudzone, wydzielają neuroprzekaźniki, dzięki czemu komórki nerwowe produkują większą ilość białek do tworzenia nowych połączeń synaptycznych lub ich stabilizacji. Brak wymienionych emocji w procesie nauki powoduje odwrotny skutek neurologiczny, czego efektem jest spadek efektywności uczenia się i w konsekwencji - zanik motywacji (Iluk 2013: 67). ${ }^{2}$

Die beim Lernen erlebten positiven Emotionen gelten als „enorm wirksame »Lernkraftverstärker «, [sie] fokussieren die Aufmerksamkeit" (Heckmair, Michl 2011: 37- 38), beeinflussen positivere Selbsteinschätzung und erhebliche Erfolgszuversicht sowie die Bereitschaft, das Wissen zu erweitern und auszudifferenzieren (Iluk 2006a: 277; Edlinger, Hascher 2008: 59-60; Sambanis 2013: 27). Bei negativen Emotionen, insbesondere unter sehr großem Druck oder Angst, werden dagegen die Aufmerksamkeitsfokussierung und damit effektives Lernen beeinträchtigt (Sambanis 2013: 27). Iluk (2006a: 274-275) stellt fest, dass negative Emotionen zu alltäglichen Erfahrungen der Lerner gehören und kognitive Kapazitäten binden, die für die Aufgabenbe-

\footnotetext{
2 ,Begeisterung und verwandte Emotionen, wie Faszination, Neugierde, Freude, Wissenshunger, sind - nach Ansicht von Neurowissenschaftlern - die wichtigste Antriebskraft für das Gehirn, weil sie die emotionalen Zentren aktivieren, die Neurotransmitter stimulieren, sodass Nervenzellen mehr Proteine produzieren, um neue synaptische Verbindungen herzustellen oder sie zu stabilisieren. Der Mangel an den genannten Emotionen im Lernprozess führt zu einem umgekehrten neurologischen Effekt, infolgedessen nimmt die Lerneffektivität ab und damit verschwindet die Motivation“ (übersetzt von M.J.).
} 
arbeitung notwendig sind. Solche Affekte „führen zu Herabsetzung intellektueller Leistungen, Beeinträchtigung der Verstehensfähigkeit, zu einer einfachen, rigiden Bearbeitung des Lernstoffes oder zu einem aktiven Fluchtoder Vermeidungsverhalten“ (Iluk 2006a: 278). Obwohl sich schon Kleinkinder der starken Auswirkung von negativen Emotionen auf das Ausbleiben kognitiver Leistungen bewusst sind, sind sie allerdings nicht in der Lage, diesen Einfluss der negativen Gefühlsregung zu neutralisieren bzw. ihm wirksam entgegenzuwirken (Iluk 2006a: 276).

Hervorzuheben ist, dass Kinder ihre Emotionen mit ungewöhnlicher Intensität und Lebhaftigkeit erleben und sie daher ein großes Bedürfnis haben, das zu lernen, was sie emotional bewegt, freut und fasziniert. Deshalb sollten diese positiven Affekte bei der frühen Fremdsprachenvermittlung berücksichtigt werden (Iluk 2002a: 134; Iluk 2002b: 96). Die kleinen Lernenden drücken ihre Emotionen mannigfaltig aus. Während einige Kinder zur negativen Selbstbewertung neigen, weisen andere Kinder ein großes Selbstbewusstsein auf. Einerseits zeigt das Kind spontan seine Freude, die durch den Kontakt mit etwas Neuem entsteht, andererseits Langeweile, die aus didaktischen Fehlern resultieren kann (Stasiak 2000: 415). Stasiak (2000: 426) unterstreicht auch, dass aufgeschlossene Kinder, die ihren Emotionen freien Lauf lassen und am Unterrichtsgeschehen aktiv beteiligt sind, den verschlossenen Kindern in den Lernleistungen und der Kreativität weitgehend überlegen sind. Im frühen Fremdsprachenerwerb zeichnen sich die Kinder durch die sog. affektive Unvoreingenommenheit aus, die mehrere Faktoren umfasst:

-kulturelle Offenheit: die Kinder verfügen noch nicht über ein starres Einstellungs- und Wertesystem, deshalb sind sie gegenüber Fremdsprachen und fremden Kulturen neutral bzw. positiv eingestellt (Leopold-Mudrack 1998: 41),

- geringe Sprechhemmungen: die Kinder sind bereit, fremdsprachliche Äußerungen spontan zu formulieren; im Gegensatz zu erwachsenen Lernenden haben sie keine Angst, durch sprachliche Fehler ihr Ansehen in der Gesellschaft zu verlieren (Arabski 1996: 84; LeopoldMudrack 1998: 39),

- problemlose Rollenübernahme: die Kinder schlüpfen gern in eine andere Rolle hinein, weil dadurch eine Identifikation mit fremdsprachlichen Sprechern stattfinden kann (Leopold-Mudrack 1998: 40),

- keine psychologische Distanz zum Zielsprachenland: die Kinder sind gegenüber den Sprechern der Zielsprache positiv eingestellt, was den Zweitsprachenerwerb spürbar fördern kann. Die Aufnahmebereitschaft für alles Neue lässt sich mit dem noch gering entwickelten Sprachbe- 
wusstsein der Kinder erklären (Arabski 1996: 84; Leopold-Mudrack 1998: 39-40; Stasiak 2000: 412),

- kindliche Spontaneität: die kindliche Unbefangenheit zeigt sich auch im spontanen Verhalten der Kinder (Leopold-Mudrack 1998: 41),

- Empathievermögen der Kinder: darunter versteht man die „Fähigkeit, sich in die Einstellungen anderer Menschen einzufühlen und zu prüfen, welche Verhaltensformen sich unter gegebenen Situationsbedingungen und mit den gängigen Normen vereinbaren lassen“" (Heyd 1991: 274).

Aus methodischer Sicht wird betont, dass der frühe Fremdsprachenunterricht von kleinen Kindern mit Freude verbunden werden soll und sich deshalb an der kindlichen Alltagswelt sowie an ihren Interessen und Neigungen orientieren sollte. Der Spracherwerb soll auch einen ganzheitlichen Lernprozess ermöglichen, der abwechslungsreich ist sowie Freude am Unterricht in den Vordergrund rückt (Kirsch 1992: 75). Das Lernen bedarf für den Aufbau von positiver, intrinsischer Motivation der Freude am Lernen. Auch Üben kann Spaß bereiten, und Wiederholung muss nicht zwangsläufig mit Langeweile assoziiert werden (Leopold-Mudrack 1998: 16; Iluk 2012a: 153). Der Unterricht sollte das Kind dazu bewegen, Neues zu entdecken, Neues auszuprobieren und Zusammenhänge nachzuvollziehen. Die Lerninhalte sind deshalb so auszuwählen, dass sie bei jüngeren Lernern Interesse Betroffenheit auslösen bzw. sie zur emotionalen Auseinandersetzung herausfordern (Iluk 2002a: 134; Iluk 2002b: 96). Dadurch kann nachweislich Motivation aufgebaut und erhalten werden, was wiederum zu einer längeren Konzentrationsfähigkeit beiträgt. Ohne Interesse und Aufmerksamkeit gibt es nämlich keine hinreichende kognitive Verarbeitung. Es gilt ebenfalls, gefühlsmäßige Beziehungen aufzubauen und für eine angenehme, angstfreie und entspannte Atmosphäre zu sorgen, die die Lernbereitschaft der Kinder beeinflusst (Leopold-Mudrack 1998: 54, 93; Iluk 2002a: 134; Iluk 2002b: 96). Im vertrauensvollen Lernklima können sich die Kinder mit fremden Inhalten und einer fremden Sprache auseinandersetzen und sie positiv erleben: „Das Lernklima der Grundschule ist ausgerichtet auf Ermutigung, Förderung und positive Lernerfahrung. In dieser Lernatmosphäre fällt es allen Kindern leicht, sich in die fremde Sprache einzuhören und mit Freude und Gewinn teilzunehmen“ (Gompf 1991: 23).

Eine Möglichkeit, die Lerninhalte so lebendig zu gestalten, dass die Kinder möglichst enge emotionale Beziehungen zum Lernstoff aufnehmen, bietet der narrative Ansatz im frühen Fremdsprachenunterricht, in dessen Mittelpunkt immer auch die Freude und die Faszination von jungen Lernern beim Zuhören stehen. 


\section{DER NARRATIVE ANSATZ IM FRÜHEN FREMDSPRACHENUNTERRICHT}

Der narrative Ansatz (story telling approach) ist darauf ausgerichtet, Kindern die Fremdsprache über entsprechend ausgewählte Geschichten zu vermitteln (Leopold-Mudrack 1998: 117; Iluk 2006b: 57-58). Als primäres Lernziel gilt nicht mehr die kommunikative Kompetenz in der Fremdsprache, sondern die Ausbildung einer Verstehenskompetenz. Der Unterricht folgt aber keiner grammatischen Progression (Kirsch 1992: 71), das Kind erlebt stattdessen ein ganzheitliches Sprachbad und hat

zumindest kurzzeitig das Erlebnis, dem Medium der fremden Sprache voll ausgesetzt zu sein; er wird angestoßen, seinen eigenen Verstehensprozess zu reflektieren [...], und er erkennt, dass sein Weltwissen sowie parasprachliche Mittel und mediale Hilfen des Lehrers ausreichen, um einen Teil der Botschaft zu entziffern (Kubanek-German 1993: 52).

Dabei werden „Wortschatz und Strukturen (...) in immer verschiedenen Situationen erfahren und damit vielfältiger vernetzt und behalten“ (Bleyhl 1996: 346). Die rechte Hemisphäre und das episodische Gedächtnis werden dann so stark aktiviert, dass das natürliche Spracherwerbspotential wirksamer genutzt wird (Kirsch 1992: 74; Schmid-Schönbein 2008: 92; LundquistMog, Widlok 2015: 131).

Im narrativen Ansatz lässt man die Kinder nicht gleich reproduktiv sprechen, so wie das in traditionellen Sprachkursen für junge Lerner normalerweise der Fall ist, in denen die sofortige Reproduktion des Lernstoffes dem Festigen und der Internalisierung des Lernstoffes dienen soll. Im narrativen Ansatz gibt man den Kindern zunächst die Gelegenheit, den Gebrauch der Sprache in sinnvollen Kontexten in Verbindung mit nonverbalem Handeln über einen längeren Zeitraum zu beobachten. Es gibt also keinen Zwang zur sofortigen Sprachproduktion (Iluk 2012b: 73). Durch den inhaltlich bedeutungsvollen Lernstoff und die verlängerte Inkubationszeit können die kleinen Lernenden ihre Aufmerksamkeit auf den Inhalt richten, Hypothesen aufstellen und sie verifizieren. Die Informationslücken innerhalb der Geschichten können die Kinder zu eigenen Denkprozessen anregen, wodurch die Phantasie und die Fähigkeit zu eigenen Ideen und Lösungsmöglichkeiten gefördert werden (Leopold-Mudrack 1998: 121). Dadurch sind sie in der Lage, die sog. kritische Masse an lexikalischen Einheiten bzw. an Spracherfahrungen zu erreichen, die für einen weiteren, beschleunigteren und effektiveren Spracherwerb unentbehrlich ist (Iluk 2011: 224).

Von Bedeutung ist ebenfalls, dass Fremdsprachenlernen stark mit sprachbegleitender Bewegung einhergehen muss. Die körperlichen Bewe- 
gungen sollten in sinnvoller Beziehung zum Erzählten / Gesagten stehen, für die Kinder nachvollziehbar sein und ihnen Vergnügen bereiten (Piepho 2002: 20-23; Iluk 2006b; Iluk 2012a; Iluk 2015: 28-34).

Geschichten sollten nach Ansicht von Iluk (2012a: 157) sowie LundquistMog und Widlok (2015: 131) im frühen Fremdsprachenunterricht ihren festen Platz finden, weil sie

an der kindlichen Erlebniswelt orientierte Inhalte bieten, wodurch sich die Kinder direkt angesprochen fühlen, einen qualitativ hochwertigen Input liefern, der sich durch einen umfangreicheren und differenzierteren Wortschatz kennzeichnet, sinnvolle und plausible Handlungszusammenhänge vermitteln, die den Lernenden die Möglichkeit bieten, sich mit der neuen Lexik beim Hören vertraut zu machen und deren Bedeutung in kontextuell transparenten Situationen zu erschließen, authentische feste, formelhafte Wendungen enthalten, die als Einheit, als so genannte "Chunks" auswendig gelernt, ohne dass sie analysiert und zusammengesetzt werden, die Lernenden dazu anregen, sprachliche Inhalte ganzheitlich (intuitiv) zu erfassen, d.h. nicht isolierte Wörter und grammatische Strukturen, sondern dem Gehörten Informationen und Intentionen zu entnehmen, das logische Denken der Kinder dadurch fördern, dass sie die dargestellten Handlungsabläufe, Zusammenhänge bzw. Ursache-Folge-Relationen interpretieren, den rezipierten Input anhand von Hypothesen in das schon internalisierte Wissensnetz einordnen; die wahrgenommenen Informationen werden in einen Gesamtzusammenhang gebracht, wodurch der Input tiefer und dauerhafter verarbeitet wird, durch die besonderen Merkmale der Narration, wie z.B. langsamere Sprechgeschwindigkeit, gestaltendes Sprechen, klare Segmentation von Wörtern und Silben ermöglichen, unbekannte Klangbilder, Sprachmuster, Sprachregeln besser zu erfassen, optimale Bedingungen für intuitiv-entdeckendes Lernen schaffen.

\section{AFFEKTIVE FAKTOREN IM NARRATIVEN ANSATZ}

Im Studienjahr 2014/2015 wurde unter der Schirmherrschaft des Instituts für Germanische Philologie der Schlesischen Universität in Katowice ein Forschungsprojekt durchgeführt, das durch das polnische Ministerium für Hochschulwesen und Wissenschaft finanziell unterstützt wurde. Um zu ermitteln, wie effektiv das Fremdsprachenlernen im Kindergarten und Primarbereich unter Einsatz des narrativen Ansatzes sein kann, wurden in drei Kindergärten und einer Grundschule Sprachkurse angeboten, in denen Deutsch als Fremdsprache nach den Prinzipien des narrativen Ansatzes unterrichtet wurde. Am Projekt haben insgesamt 95 Kinder 65 Kindergarten- 
und 30 Grundschulkinder) teilgenommen. Unter methodischer Aufsicht von 5 Tutoren wurde der Deutschunterricht von 11 Lehramtsstudierenden aus dem Masterstudiengang vom 16. Februar bis zum 20. Juni 2015 erteilt.

\subsection{Aufbau der Sprachkurse}

Der Sprachkurs umfasste in jeder Kindergruppe insgesamt 38 Unterrichtsstunden. Angesichts der geringen Konzentrationsfähigkeit der Kindergartenkinder beschränkte sich der Deutschunterricht wöchentlich auf zwei Unterrichtseinheiten zu je 35-40 Minuten. Die Kinder im Grundschulalter erhielten dagegen Deutschunterricht in zwei Blöcken wöchentlich zu je 45 Minuten.

In allen Projektgruppen wurden 5 narrative Texte eingesetzt: Rübenziehen; Die kleine Raupe Nimmersatt; In einem dunklen, dunklen Wald; Kim und eine kleine Hexe; Die drei kleinen Schweinchen. Für die Auswahl dieser Märchen war es entscheidend, dass diese Texte u.a. durch eine hohe lexikalische Redundanz gekennzeichnet sind, sich wiederholende Textpassagen enthalten sowie die Animation des Inhalts ermöglichen. Bevor sie aber eingesetzt wurden, wurden sie entsprechend adaptiert und lexikalisch vereinfacht.

Die Arbeit mit einem Märchen nahm etwa 4-5 Unterrichtsstunden in Anspruch. Im Falle der Kinder im Grundschulalter kamen erweiterte Versionen derselben Texte zum Einsatz. Der Inhalt des Märchens wurde mit Hilfe einer Handpuppe und entsprechender Requisiten dargeboten. Eine angemessene Visualisierung (Bilder, Arbeitsblätter, Mimik, Gestik) half den Kindern, neue Wörter und Wendungen in der Fremdsprache zu erschließen. In einigen Fällen konnten die Kinder vor der Märchenpräsentation den Textinhalt nach folgendem Muster antizipieren; wenn nötig auch in der Muttersprache (Iluk 2012b: 68-69):

- die Protagonisten einer Geschichte,

- die relevantesten Gegenstände und ihre Merkmale,

- die relevanten Handlungen,

- die Handlungsplätze, Handlungszeiten und Handlungsmodalitäten.

Mit dem Experiment galt es unter anderem zu überprüfen, ob der narrative Ansatz die intrinsische Motivation, die Selbstwirksamkeit, die Vorstellungskraft und Kreativität der Kinder aufbauen hilft. ${ }^{3}$

\footnotetext{
${ }^{3}$ Mit dem Forschungsprojekt galt es vor allem nachzuweisen, ob die Kinder in der Lage sind, ein relativ hohes und stabiles Verstehensvermögen (an der Anzahl von Vokabeln und Sätzen gemessen) zu erreichen und ob sie die Fähigkeit erwerben, auch längere fremdsprachliche Äußerungen zu verstehen und grammatische Strukturen korrekt zu verarbeiten. Die Test-
} 


\subsection{Messinstrumente}

Zur Überprüfung der aufgestellten Forschungshypothesen wurden solche Messinstrumente entwickelt, bei denen besonderer Wert auf eine kindgerechte Präsentation gelegt wurde, wobei die eigentliche Erhebung aus drei getrennten Teilen bestand.

Im ersten Teil wurde das Multiple-Choice-Verfahren eingesetzt. Den Kindergartengruppen wurden 15 Bilderfolgen angeboten, den Grundschulgruppen 18 Bilderfolgen, die jeweils aus 4 Illustrationen bestanden. Die Kinder hörten Sätze (und nicht einzelne Wörter!) und mussten nach dem Hören jeden Satz einem von vier Bildern zuordnen. Jeder Satz wurde vom Testleiter jeweils zweimal vorgelesen.

Der zweite Teil orientierte sich an der Total Physical Response-Methode (Lovik 1996; Klippel 2000). ${ }^{4}$ Jedem Kind wurde eine Reihe von 10 Anweisungen vorgegeben, auf die es entsprechend reagieren sollte, indem es eine Handlung ausführte, etwas gestisch und mimisch vorführte oder eine Zeichnung anfertigte. Für die Auswahl dieser Testform war die Tatsache bedeutsam, dass die Kinder durch die auszuführenden Bewegungen oder Handlungen von der Testsituation abgelenkt wurden, aber dabei gleichzeitig ihr Verstehen von typischen Anweisungen überprüft werden konnte.

Im dritten Teil wurden den Probanden Sätze zu Bildern angeboten, die entweder den Inhalt der Bilder wiedergaben oder andere Dinge beschrieben. Im anschließenden Testteil wurden den Probanden insgesamt 6 Sätze vorgesprochen, die von ihnen mit "richtig" oder "falsch" eingestuft werden mussten. Aufgrund des Rätselcharakters von True-false-Statements beteiligten sich die Kinder an dieser Art von Aufgaben mit großem Engagement.

\subsection{Ergebnisse der Evaluation}

Um die Richtigkeit der Annahme zu belegen, dass der narrative Ansatz die intrinsische Motivation, die Selbstwirksamkeit, die Vorstellungskraft

ergebnisse der Kindergarten- und Grundschulkinder, die in beiden Effizienzmessungen gewonnen wurden, wurden im Beitrag von Iluk und Jakosz (2017) ausführlich dargestellt.

${ }^{4}$ Total Physical Response ist eine Fremdsprachenvermittlungsmethode, die in den sechziger Jahren von dem Lernpsychologen James Asher in den Vereinigten Staaten entwickelt wurde. Die Lehrenden geben den Lernenden eine Reihe von Anweisungen in der Fremdsprache vor. Die Lernenden zeigen, dass sie die Anweisung verstanden haben, indem sie sie ausführen. Zunächst agiert aber die Lehrkraft als Modell, d.h. führt eine Anweisung selbst aus und die Lernenden schauen nur zu. Im weiteren Schritt imitieren die Lernenden zusammen mit der Lehrkraft die Handlungen. Später gibt die Lehrkraft eine Anweisung vor, ohne sie selbst auszuführen. Die Lernenden agieren jetzt allein (Lovik 1996: 41-42; Klippel 2000: 256). 
und Kreativität der Kinder aufzubauen hilft, wurden Videoaufzeichnungen und Beobachtungsnotizen der Tutoren sowie die Meinungen der Kursleiter einer Analyse unterzogen.

Im Rahmen des Sprachunterrichts war immer wieder zu bemerken, dass der narrative Ansatz die Motivation und Kreativität aller Kindergruppen weckte sowie ihre Selbstwirksamkeit beeinflusste. Dies war zum großen Teil dadurch möglich, dass die Märchen einen besonders starken Anreiz zur Sprachverarbeitung gaben. Die präsentierten Inhalte, aber auch die lockere Lernatmosphäre sowie die spezifischen Ausdrucksmittel, darunter Reime, Alliterationen und zahlreiche Textwiederholungen sowie gestaltendes Erzählen halfen den Kindern, den Lernstoff direkt über die Fremdsprache zu erschließen und sich diese dauerhaft anzueignen.

\subsubsection{Handpuppe als emotionaler Ansprechpartner}

Aus psychologischen und emotionalen Gründen ist der Einsatz einer Handpuppe im frühen Fremdsprachenunterricht wichtig, denn diese "kommt" aus einem anderen Land und vermittelt als ein Vertreter der fremdsprachlichen Kultur ausschließlich in einer fremden Sprache zwischen der Lehrkraft und den kleinen Lernenden, was für einen wirklichen Informationsaustausch in einem für die Kinder bedeutungsvollen Kontext sorgt und einen wertvollen Beitrag zur weitgehenden Einsprachigkeit des Fremdsprachenunterrichts leistet (Maier 1991: 194; Haudeck 2000: 59-60; Iluk 2006b: 86; Schmid-Schönbein 2008: 68-69):

Einerseits wird sie ein Mitglied der Lerngemeinschaft: Die Kinder werden allmählich ihre Lebensgeschichte entdecken und ihren Charakter kennenlernen, so wie die Puppe gleichzeitig die Kinder kennenlernt. In dieser Phase wird die Puppe zum Partner, der die Kinder so weit versteht, dass er für sie sprechen kann. Die Puppe informiert die Lehrkraft, was in der Lerngruppe passiert (ein Kind fehlt heute, ein Kind hat Geburtstag, die Kinder sind heute sehr müde, weil sie letzte Nacht ein Fußballspiel geguckt haben). Andererseits aber wirkt die Puppe auch als Lehrkraft, denn sie gibt Sprachmuster vor und flüstert Wörter ins Ohr (Legutke 2006: 8).

Da die Handpuppe die Kinder weder benotet noch ermahnt, lassen sich Sprechhemmungen besonders bei schüchternen Lernanfängern abbauen. Schrittweise trauen diese sich, die Fremdsprache als Kommunikationsmittel anzuwenden, weil die Aufmerksamkeit der Klasse stärker auf die Handpuppe und weniger auf sie selbst gerichtet ist (Haudeck 2000: 59-60; Palmer 2008: 9; Stahl-Morabito 2012: 40). Die Handpuppe steht den Kindern nah, ist 
für sie wie ein Freund und spricht sie emotional an: Die jungen Lernenden können sie nämlich knuddeln, an sich drücken und zu ihr sprechen (Iluk 2006b: 86; Schmid-Schönbein 2008: 68; Iluk 2015: 27, Lundquist-Mog, Widlok 2015: 144).

Aus unseren Beobachtungen ist zu schließen, dass die Kinder ungeduldig auf jeden Deutschunterricht warteten. Sie nahmen mit sichtlichem Vergnügen daran teil, interagierten gern mit der Handpuppe und hörten mit starkem Interesse zu. Dieser emotionale Bezug wirkte sich positiv auf die Lernmotivation und die Wahrnehmungsprozesse aus, die ohne übermäßigen kognitiven Aufwand abliefen und den Kindern viel Spaß bereiteten. Die Kursleiter bestätigten, dass die Handpuppe ein unentbehrlicher Bestandteil eines jeden Deutschunterrichts war und jeder Verzicht auf ihren Einsatz im Unterricht von Kindern als deutlich negativ empfunden wurde. Zu Unterrichtsbeginn begrüßte die Handpuppe die Lernenden und fragte nach ihrem Befinden. Die Kinder beantworteten sehr willig ihre Fragen und folgten ihren Anweisungen. Es kam auch manchmal vor, dass eines der Kinder den Kursleitern die Handpuppe wegnahm und mit ihrer Hilfe solche Fragen wie »Wie heißt du? « und »Wie geht es dir? « an andere Kinder stellte, ohne dazu aufgefordert zu werden. Hervorhebung verdient noch eine Situation, in der ein verschlossenes Mädchen eines Tages sehr traurig war und sich weder von einer Kursleiterin noch von anderen Kindern trösten ließ. Sobald aber die Handpuppe erschien, strahlte es vor Freude, drückte es sie stark an sich und äußerte den Wunsch, mithilfe der Handpuppe die anderen Kinder zu begrüßen. Im weiteren Verlauf des Unterrichts waren schon keine Anzeichen seiner früheren Trauer mehr zu beobachten.

\subsubsection{Freude am Lernen mit Bewegung}

Die unter Punkt 2 erwähnten positiven Lerneffekte durch den Einsatz der Bewegung im Unterricht konnten in diesem Projekt bestätigt werden. Es wurden solche Bedingungen geschaffen, die es ermöglichten, Sprache und Handeln in Verbindung zu bringen. Dies geschah u.a. während der Animation der Märchenfiguren durch die Kinder sowie in anschließenden Bewegungsspielen zur Festigung der Redemittel. Damit kamen wir dem starken Bedürfnis der Kinder nach Bewegung und handelndem Spielen entgegen. Die zum Inhalt des Textes oder einer Aufgabe ausgeführte Handlung signalisierte darüber hinaus dem Lehrer, wie das jeweilige Kind den Input verstanden hat und den anderen Kindern bot die Beobachtung des handelnden Kindes die Gelegenheit zur Überprüfung eigener Bedeutungshypothesen. 
Die Kinder beteiligten sich gern an vorgeschlagenen Bewegungsübungen, weil der Sinn der Lernhandlungen für sie einsichtig, deren Ausführung herausfordernd war und ihnen Freude bereitete.

\subsubsection{Einfluss der verlängerten Aperzeptionsphase auf die Lernbereitschaft}

Im frühen Fremdsprachenunterricht sollten die Kinder nicht zum Sprechen gezwungen werden. Ähnlich wie im Erstsprachenerwerb sollten sie mit möglichst viel authentischer Sprache konfrontiert werden und dabei Gelegenheit bekommen, die Sprache in einer Phase des Zuhörens zu rezipieren und zu verarbeiten, bevor sie sprachlich produktiv werden (Legutke et al. 2013: 291). Sie müssen sich "durch den Hof des passiven Wortschatzes durcharbeiten“ (Bleyhl 1995: 26), „wo entscheidende mentale Prozesse der Sprachverarbeitung und -rekonstruktion sowie ihre Internalisierung stattfinden" (Iluk 2012a: 153). Die Kinder können aber das Verständnis fremdsprachlicher Äußerungen z.B. durch Gestik und Mimik signalisieren.

Der fehlende Druck zu sofortiger Reproduktion von noch nicht hinreichend internalisierten fremden Sprachstrukturen beeinflusste die Bereitschaft der Kinder positiv, die gehörten fremdsprachigen Äußerungen selbstständig zu erschließen sowie deren dauerhaftes Memorieren. Dies ist nicht der Fall im sog. traditionellen fremdsprachlichen Frühunterricht. Iluk (2008: 55-56) konnte nämlich im Rahmen anderer Kindergartenexperimente feststellen, dass Versuche, die Kinder von der ersten Stunde an zum Sprechen in der Fremdsprache zu mobilisieren, fehlschlugen. Mehr noch: Die Kinder verloren nicht nur sehr schnell ihr Interesse an den reproduktiven (imitativen) Sprechübungen, sondern generell am Fremdsprachenlernen. Der Grund hierfür liegt darin, dass Kinder bei gleichzeitiger Entwicklung des Hörverstehens und der Sprechfertigkeit in der Anfangsphase kognitiv überfordert werden, was an der Senkung ihres Konzentrationsgrades sowie und der starken Anfälligkeit für Ablenkungsreize leicht erkennbar ist (Iluk 2012b: 73-74). Diese Probleme beobachtete man dagegen bei Kindern, die nach den Prinzipien des narrativen Ansatzes unterrichtet wurden, nicht bzw. nur im minimalen Ausmaß.

Im Verlauf des ganzen Sprachkurses zeichneten sich die Kinder durch eine große Leistungsbereitschaft und Ausdauer aus. Es herrschte ein entspanntes Lernklima, dennoch waren die Kinder konzentriert und aktiv. Sie reagierten und antworteten anfänglich auf den sprachlichen Impuls ohne Rückgriff auf zielsprachliche Mittel, sondern bewiesen durch das Ausführen von Handlungen ihr Verständnis der Bedeutung von Wortschatz oder Struk- 
turen, was laut Sambanis (2005: 9) deren Verstehens- und Behaltensleistung erhöht. Schon nach zwei Monaten (Mai/Juni 2015) konnte man immer häufiger beobachten, wie sich einzelne Kinder freiwillig und schrittweise in die Narration der präsentierten Märchen einschalteten. Der Lehrer erzählte die Geschichte und an der Stelle, an der er die Narration unterbrach, ergänzten die Lernenden das fehlende Wort, die fehlende Phrase bzw. eine ganze Textpassage. Es galt dennoch die Regel, dass die Kinder nicht gezielt aufgefordert wurden, die entsprechenden Wörter oder Phrasen laut zu ergänzen. Jeder Lerner entschied selbst, wann er an der Narration einer Geschichte teilnehmen wollte. Die Kinder gaben an, dass sie eine große Freude empfanden, wenn sie sich am Erzählen durch Mitsprechen wiederkehrender Textpassagen, die natürliche und von ihnen durchaus akzeptierte Wiederholungsmöglichkeiten bieten, frei beteiligen konnten. Sie waren sogar in der Lage, bei minimaler Unterstützung der Projektleiterinnen ein ganzes Märchen (z.B. Rübenziehen oder In einem dunklen, dunklen Wald) nachzuerzählen. Man konnte zudem sehen, dass die Kinder sogar außerhalb des Sprachunterrichts, d.h. bei anderen Lernaktivitäten mit ihrer Erzieherin, aus eigener Initiative die Refrains der im Sprachkurs gelernten Lieder summten. Den produktiven Sprachgebrauch und dabei die positive Einstellung unserer Probanden zur gelernten Sprache bestätigt deutlich eine Situation, in der die Kinder mit ihrer Erzieherin auf einem Spaziergang waren und auf der Straße aus eigener Initiative ein deutsches Lied laut sangen, ohne sich dabei befangen zu fühlen. Die vorbeigehenden Passanten, darunter zufälligerweise die Leiterin eines Kindergartens, schauten den souverän singenden Kindern mit Sympathie und Staunen zu.

\section{FAZIT}

Unsere empirischen Befunde liefern den Beweis dafür, dass der narrative Ansatz authentische und bei Kindern beliebte Kommunikationssituationen schafft, wodurch positive Emotionen entstehen, eine starke intrinsische Lernmotivation aufgebaut und der sprachliche Input besser (d.h. ganzheitlich und intuitiv) aufgenommen und verarbeitet werden kann. Bei allen Kindern ließ sich eine sichtlich positive Haltung gegenüber der Fremdsprache und fremden Kultur aufbauen. Eine solche Einstellung leistete zugleich einen Beitrag zum positiven Lernklima, das von einem angstfreien Lernen ohne Sprechhemmungen geprägt und von Neugier, hoher Motivation und Lernbereitschaft getragen wurde.

Ein in positiver Atmosphäre verlaufender und zugleich erfolgreicher Fremdsprachenunterricht, in dem Kinder von Anfang zielsprachenorientiert 
agieren, schafft auch eine gute Voraussetzung für die dauerhafte Bereitschaft, die im Kindergarten gelernte Fremdsprache weiter zu lernen. Dies bestätigten u.a. die Kinder selbst, als sie am Kursende fragten, ob sie im nächsten Schuljahr weiter Deutsch lernen werden. Solche Wünsche sind nach einem traditionell geführten Sprachunterricht, in dem die Lerner häufig Langeweile und Monotonie erleben, eher die Ausnahme.

\section{LITERATURVERZEICHNIS}

Arabski, J. (1996). Uwarunkowania neurolingwistyczne i wiek krytyczny. In: J. Arabski (Hrsg.). Przyswajanie języka obcego i pamięć werbalna. Katowice: Wydawnictwo Uniwersytetu Śląskiego, S. 64-86.

Bleyhl, W. (1995). Wortschatz und Fremdsprachenunterricht oder: Das Problem sind nicht die Lerner. In: K.-R. Bausch / H. Christ / F.G. Königs / H.-J. Krumm (Hrsg.). Erwerb und Vermittlung von Wortschatz im Fremdsprachenunterricht. Tübingen: Gunter Narr, S. 20-31.

Bleyhl, W. (1996). Der Fallstrick des traditionellen Lehrens und Lernens fremder Sprachen. Vom Unterschied zwischen linearem und nicht-linearem Fremdsprachenunterricht. Praxis des neusprachlichen Unterrichts, 4, S. 339-347.

Bleyhl, W. (2003). Grammatikunterricht in der Grundschule? Fremdsprachen Frühbeginn (FF), 4, S. 5-9.

Ciompi, L. (1997). Die emotionalen Grundlagen des Denkens. Entwurf einer fraktalen Affektlogik. Göttingen: Vandenhoeck \& Ruprecht.

Edlinger, H. / Hascher, T. (2008). Von der Stimmungs- zur Unterrichtsforschung: Überlegungen zur Wirkung von Emotionen auf schulisches Lernen und Leisten. Unterrichtswissenschaft, 36 (1), S. 55-70.

Gładysz, J. (2007). Empirische Untersuchung der Effizienz des narrativen Ansatzes. In: E. Białek / C. Lipiński / E. Tomiczek (Hrsg.). Orbis Linguarum, Vol. 32. Wrocław: Oficyna Wydawnicza ATUT, S. 205-230.

Gompf, G. (1991). Didaktische Ansätze des Frühlernens von Fremdsprachen. In: K. Ermert (Hrsg.). Loccumer Protokolle. Frühes Fremdsprachenlernen - Schulreform für Europa. 1. Auflage. Loccum: Evangelische Akademie Loccum, S. 22-28.

Haudeck, H. (2000). Der Einsatz von Handpuppen und anderen Spielfiguren. In: W. Bleyhl (Hrsg.). Fremdsprachen in der Grundschule. Grundlagen und Praxisbeispiele. Hannover: Schroedel, S. 59-68.

Heckmair, B. / Michl, W. (2011). Bewegung und Erlebnis als Nährboden des Lernens. DIE Zeitschrift für Erwachsenenbildung: Lernen in Bewegung, 1, S. 37-40.

Heyd, G. (1991). Deutsch lehren - Grundwissen für den Unterricht in Deutsch als Fremdsprache. Frankfurt am Main: Diesterweg.

Iluk, J. (2002a). Befähigung zum Ausdruck von Emotionen in der Fremdsprache als Lernziel. Linguistica Silesiana, 23, S. 133-142.

Iluk, J. (2002b). Probleme der Befähigung zum Ausdruck von Emotionen in der Fremdsprache aus curricularer Sicht. Deutsch als Fremdsprache, 39 (2), S. 96-102.

Iluk, J. (2005). Methodische Binsenweisheiten und der alltägliche Fremdsprachenunterricht. In: H. Pürschel / T. Tinnefeld (Hrsg.). Moderner Fremdsprachenerwerb zwischen Interkulturalität und Multimedia. Reflexionen und Anregungen aus Wissenschaft und Praxis. Bochum: AKSVerlag, S. 164-174. 
Iluk, J. (2006a). Emotionale Aspekte des (Fremdsprachen)Lernens. In: Z. Mielczarek / G. Kowal (Hrsg.). Erinnerte Zeit. Festschrift für Lothar Pikulik zum 70. Geburtstag. Częstochowa: Wydawnictwo Wyższej Szkoły Lingwistycznej, S. 273-281.

Iluk, J. (2006b). Jak uczyć małe dzieci języków obcych? Częstochowa: Wydawnictwo Wyższej Szkoły Lingwistycznej.

Iluk, J. (2008). Teoria i praktyka w nauczaniu języków obcych w przedszkolu. Jezzyki Obce $w$ Szkole, 4, S. 48-59.

Iluk, J. (2011). Der narrative Ansatz im frühen Fremdsprachenunterricht und seine Effizienz. In: S. Adamczak-Krysztofowicz / M. Kowalonek-Janczarek / M. Maciejewski / A. Sopata (Hrsg.). Aktuelle Probleme der Angewandten Linguistik. Interkulturalität als Schlüsselkompetenz für Fremdsprachenlehrer, Übersetzer und Mediatoren. Poznań: Wydawnictwo Naukowe UAM, S. 217-225.

Iluk, J. (2012a). Der narrative Ansatz und dessen Effizienz im Kindergarten und im Primarbereich. Deutsch als Fremdsprache, 49 (3), S. 150-159.

Iluk, J. (2012b). Praktische Anweisungen zum narrativen Ansatz im fremdsprachlichen Frühunterricht. In: M. Bujňáková / J. Paračková / Ch. Irsfeld (Hrsg.). Deutsch in Forschung und Lehre. Teil I, Sammelband, X. Tagung des Verbandes der Deutschlehrer und Germanisten der Slowakei (1.-4. September 2010, Prešov). Prešov: Filozofická fakulta Prešovskej univerzity, S. 65-75.

Iluk, J. (2013). Jak (de)motywujemy uczniów na lekcjach języka obcego? Języki Obce w Szkole, 4, S. 67-74.

Iluk, J. (2015). Przygotowanie dzieci do posługiwania się językiem obcym w kontekście nowego rozporządzenia Ministerstwa Edukacji Narodowej. In: J. Iluk (Hrsg.). Nauczanie języków obcych w przedszkolu i na etapie wczesnoszkolnym na przykładzie języka niemieckiego. Katowice: Wydawnictwo Uniwersytetu Śląskiego, S. 15-36.

Iluk, J. / Jakosz, M. (2017). Narrativer Fremdsprachenunterricht im Vor- und Schulalter aus der Perspektive der interkulturellen Erziehung. In: A. Kątny (Hrsg.). Studia Germanica Gedanensia 37. Band: Kontrastive Linguistik und Interkulturelle Kommunikation: Sprach- und Kulturkontakte. Gdańsk: Wydawnictwo Uniwersytetu Gdańskiego, S. 111-128.

Kirsch, D. (1992). Der narrative Ansatz im frühen Fremdsprachenunterricht. In: GoetheInstitut/The British Council/ENS-Credif (Hrsg.). Fremdsprachenlernen in der Grundschule. Triangle 11. Paris: Didier Erudition, S. 71-77.

Klippel, F. (2000). Englisch in der Grundschule: Handbuch für einen kindgemäßen Fremdsprachenunterricht. Berlin: Cornelsen.

Krashen, S.D. (1985). The Input Hypothesis: Issues and Implications. New York: Longman.

Kubanek-German, A. (1993). Zur möglichen Rolle des Narrativen im Fremdsprachenunterricht für Kinder. Primar: Zeitschrift für Deutsch als Fremdsprache im Primarbereich, 3, S. 48-54.

Legutke, M. (2006). Die Handpuppe als Partner. Ein hilfreiches Medium im Unterricht. Primary English. Die Fachbeilage Grundschulmagazins Englisch, 6, S. 8-9.

Legutke, M. / Schocker-von Ditfurth, M. / Müller-Hartmann, A. (2013). Frühbeginnender Fremdsprachenunterricht. In: W. Hallet / F.G. Königs (Hrsg.). Handbuch Fremdsprachendidaktik. Seelze-Velber: Kallmeyer / Klett, S. 290-293.

Leopold-Mudrack, A. (1998). Fremdsprachenerziehung in der Primarstufe: Voraussetzungen, Konzept, Realisierung. Münster et al.: Waxmann.

Lovik, Th.A. (1996). Total Physical Response: Beschreibung und Beurteilung einer innovativen Methode. FluL, 25, S. 38-47.

Lundquist-Mog, A. / Widlok, B. (2015). DaF für Kinder. München: Klett-Langenscheidt. 
Maier, W. (1991). Fremdsprachen in der Grundschule. Eine Einführung in ihre Didaktik und Methodik. Berlin: Langenscheidt.

Palmer, S. (2008). Mein Partner - die Handpuppe. "ELAAN": Magazin für Lehramtsanwärter/innen, Magazin für Referendare/innen/. Verband Bildung und Erziehung, Landesverband NRW, 38, S. 9-11.

Peterßen, W.H. (1999). Kleines Methoden-Lexikon. München: Oldenbourg.

Piepho, H.-E. (2002). Stories' ways. In: W. Bleyhl (Hrsg.). Fremdsprachen in der Grundschule. Geschichten erzählen im Anfangsunterricht. Storytelling. Hannover: Schroedel, S. 20-34.

Sambanis, M. (2005). Verstehensbasierte Ansätze im frühen Fremdsprachenunterricht - Weg oder Irrweg? Beiträge zur Fremdsprachenvermittlung, 43, S. 3-11.

Sambanis, M. (2013). Fremdsprachenunterricht und Neurowissenschaften. Tübingen: Narr Francke Attempto.

Schmid-Schönbein, G. (2008). Didaktik und Methodik für den Englischunterricht. Kompakter Überblick. Ziele - Inhalte - Verfahren. Für die Klassen 1 bis 4. Berlin: Cornelsen Verlag Scriptor.

Stahl-Morabito, N. (2012). Ein Jahr mit Bumblebee - friends at last? Zum Einsatz der Handpuppe im Englischunterricht im Förderschwerpunkt Lernen. Sonderpädagogische Förderung in NRW, 1, S. 39-43.

Stasiak, H. (2000). Wpływ czynników emocjonalnych na dziecięcą akwizycję języka obcego. In: B.Z. Kielar / T.P. Krzeszowski / J. Lukszyn / T. Namowicz (Hrsg.). Problemy komunikacji międzykulturowej. Lingwistyka, translatoryka, glottodydaktyka. Warszawa: Graf-Punkt, S. 402-427.

Vogel, K. (1993): Input und Fremdsprachenerwerb. Psycholinguistische Überlegungen zu Rolle und Funktion des sprachlichen Inputs beim Lernen und Verstehen einer Fremdsprache. Neusprachliche Mitteilungen (NM), 3, S. 151-162.

Weskamp, R. (2003). Fremdsprachenunterricht entwickeln. Grundschule - Sekundarstufe I - Gymnasiale Oberstufe. Hannover: Schroedel.

Received: 29.11.2017; revised: 28.01.2018 